Matsushima, Y.

Osaka J. Math.

16 (1979), 617-631

\title{
ON THE INTERMEDIATE COHOMOLOGY GROUP OF A HOLOMORPHIC LINE BUNDLE OVER A COMPLEX TORUS
}

\author{
Yozo MATSUSHIMA
}

(Received August 7, 1978)

Let $E=V / L$ be a complex torus, where $V$ is an $n$-dimensional complex vector space and $L$ a lattice of $V$. Let $H$ be a Hermitian form on $V$ and $A$ the imaginary part of $H$. Then $A$ is an $\boldsymbol{R}$-bilinear alternating form on $V$. We say that $H$ is a Riemann form of signature $(s, r)$ for the torus $E$ if

(a) $H$ is non-degenerate and of signature $(s, r)$;

(b) $A$ is integral valued on the lattice $L$.

To a Riemann form $H$ we associate a factor

$$
J_{H, \psi}(g, z)=\psi(g) \varepsilon\left[\frac{1}{2 i} H(z, g)+\frac{1}{4 i} H(g, g)\right]
$$

with $g \in L, z \in V$, where $\varepsilon[\cdot]=\exp 2 \pi i \cdot$ and $\psi$ is a map from $L$ to $C_{1}^{*}=$ $\{z \in \boldsymbol{C}|| z \mid=1\}$ satisfying $\psi(g+h)=\psi(g) \psi(h) \varepsilon\left[\frac{1}{2} A(g, h)\right] ;$ the function $\psi$ being called a semi-character of $L$ for $A$.

The factor $J_{H, \psi}: L \times V \rightarrow C^{*}$ satisfies the equation

$$
J_{H, \psi}(g+f, z)=J_{H, \psi}(g, h+z) J_{H, \psi}(h, z),
$$

where $g, h \in L, z \in V$.

Given the factor $J_{H, \psi}$ we define an action of the lattice group $L$ on $V \times C$ by the rule

$$
(z, \xi) \cdot g=\left(z+g, J_{H, \psi}(g, z) \xi\right),
$$

where $z \in V, \xi \in C$ and $g \in L$. The action of $L$ on $V \times C$ is free and the quotient of $V \times \boldsymbol{C}$ by $L$ has a natural structure of a holomorphic line bundle over the complex torus $E=V \mid L$. We shall denote this line bundle by $F(H, \psi)$.

The following vanishing theorem for the cohomology of $F(H, \psi)$ is wellknown [2, 4]: If $H$ is a Riemann form of signature $(s, r)$, then we have

$$
H^{q}(E, F(H, \psi))=0
$$


for $q \neq r$ (for a proof see Appendix 2 of this paper).

In particular if $r=0$, namely if $H$ is positive definite, $H^{q}(E, F(H, \psi))=0$ except for $q=0$ and $H^{0}(E, F(H, \psi))$ is identified with the space of all holomorphic theta functions on $V$ for the factor $J_{H, \psi}$. Replacing $H$ by a suitable positive interger multiple of $H$, if necessary, these theta functions define a holomorphic imbedding of $E$ into a complex projective space. A complex torus which admits a positive definite Riemann form is called an abelian variety. There exist complex tori which are not abelian varieties but which admit Riemann forms of signature $(s, r)$ with $s>0$ and $r>0$ (see Appendix 1). For such a complex torus $E$, there exists no non-trivial theta function. However, there exists the non-trivial intermediate conomology group $H^{r}(E, F(H, \psi))$ with $0<r<n$.

The purpose of this paper is to give an interpretation of the intermediate cohomology group $H^{r}(E, F(H, \psi))$. Namely we associate to a Riemann form $H$ of signature $(s, r)$ a family of polarized abelian varieties $\left(E_{b}, H_{b}\right)$ parametrized by elements $b$ of the Hermitian symmetric space $\mathcal{B}=U(H) / K$, where $U(H)$ is the unitary group of the Hermitian form $H$ and $K$ is a maximal compact subgroup of $U(H)$. Here $E_{b}$ is of the form $E_{b}=V_{b} / L$, where $V_{b}$ is an $n$-dimensional complex vector space with the same underlying real vector space as $V$ and with a complex structure $J_{b}$ distinct from that of $V$ and parametrized by $b \in \mathscr{S}$ and $H_{b}$ is a positive definite Riemann form for $E_{b}$ whose imaginary part is equal to $A$. We then assign a family of line bundles $F\left(H_{b}, \psi\right)$ over $E_{b}$ for each $b$. Finally we shall show that there exists a canonically defined isomorphism from $H^{r}(E, F(H, \psi))$ to $\left.H^{0}\left(E_{b}, F_{b}, \psi\right)\right)$ for each $b$. We also see that there exists a family $\Phi_{b}$ of differentiable imbedding of $E$ into a complex projective space which is partially holomorphic and partially antiholomorphic.

It should be mentioned that C.L. Siegel [3] has associated to an indefinite quadratic form a family of theta series parametrized by a symmetric space. It is possible to interpret Siegel's family of theta series as a subfamily of the family $\left\{H^{0}\left(E_{b}, F\left(H_{b}, \psi\right)\right)\right\}$ of theta functions attached to a certain complex torus $E$ and a Riemann form $H$ related with the given indefinite quadratic form.

\section{A Riemann form of signature $(s, r)$ and a family of polarized abelian varieties}

Let $E=V \mid L$ be a complex tours, where $V$ is an $n$-dimensional complex vector space and $L$ a lattice of $V$. We shall denote by $W$ the underlying $2 n$ dimensional real vector space of $V$ and by $J$ the complex structure of $W$ defining the complex vector space $V$.

Let $H$ be a non-degenerate Hermitian form on $V$ of signature $(s, r)$, where $s+r=n$. We denote by $A$ the imaginary part of $H$. Then we have

$$
H(u, v)=A(J u, v)+i A(u, v), u, v \in W .
$$


We assume that the alternating $\boldsymbol{R}$-bilinear form $A$ to be integral valued on $L \times L$ and we call $H$ a Riemann form of signature $(s, r)$ for the complex tours $E$.

We shall denote by $U(H)$ the unitary group of the Hermitian form $H$. $A$ basis $B=\left\{v_{1}, \cdots, v_{n}\right\}$ of $V$ is said to be a privileged basis for $H$ if the matrix of $H$ with respect to the basis $B$ is of the form

$$
1_{s, r}=\left(\begin{array}{cc}
1_{s} & 0 \\
0 & -1_{r}
\end{array}\right)
$$

where $1_{s}$ and $1_{r}$ denote the unit matrix of size $s$ and $r$ respectively.

The group $U(H)$ acts simply transitively on the set of all privileged bases for $H$. We denote by $V_{1}(B)$ and $V_{2}(B)$ the subspaces of $V$ spanned by $\left\{v_{1}, \cdots, v_{s}\right\}$ and $\left\{v_{s+1}, \cdots, v_{n}\right\}$ respectively. Then we have

$$
W=V_{1}(B) \oplus V_{2}(B) .
$$

We say that two privileged bases $B$ and $B^{\prime}$ are equivalent, $B \sim B^{\prime}$, if $V_{i}(B)=$ $V_{i}\left(B^{\prime}\right)$ for $i=1,2$. We shall denote by $\mathscr{B}$ the set of equivalence classes of priveleged bases for $H$. Then the group $U(H)$ acts transitively on $\mathscr{B}$ and $\mathscr{B}$ is identıfied with the Hermitian symmetric space $U(H) / K$, where $K$ is a maximal compact subgroup of $U(H)$.

Let $b \in \mathcal{B}$ and let $B$ be a privileged basis representing $b$. We define a linear transformation $J_{b}$ of $W$ by requiring

and

$$
J_{b}=J \text { on } V_{1}(B)
$$

$$
J_{b}=-J \text { on } V_{2}(B) \text {. }
$$

We have $J_{b}^{2}=-1$ and hence $J_{b}$ defines a complex structure on $W$. We shall denote by $V_{b}$ the complex vector space defined by $W$ and $J_{b}$.

Define the symbol $\varepsilon_{k}(k=1,2, \cdots, n)$ by

$$
\varepsilon_{k}=\left\{\begin{array}{cl}
1, & k \in[1, s], \\
-1, & k \in[s+1, n] .
\end{array}\right.
$$

If $B=\left\{v_{1}, \cdots, v_{n}\right\}$ is a privileged basis representing $b$, then we have

$$
J_{b} v_{k}=\varepsilon_{k} J v_{k}
$$

We also have $H\left(v_{k}, v_{j}\right)=\varepsilon_{k} \cdot \delta_{k j}$ and since $H\left(v_{k}, v_{j}\right)=A\left(J v_{k}, v_{j}\right)+i A\left(v_{k}, v_{j}\right)$, we get $A\left(v_{k}, v_{j}\right)=0$ and $A\left(J v_{k}, v_{j}\right)=\varepsilon_{k} \cdot \delta_{k j}$. It follows from these that the decomposition (2) is orthogonal for $A$ and also for $H$. We have also $A\left(J_{b} u, J_{b} v\right)=A(u, v)$ for $u, v \in W$. For let $u=u_{1}+u_{2}, v=v_{1}+v_{2}$ with $u_{1}, v_{1} \in V_{1}(B)$ and $u_{2}, v_{2} \in V_{2}(B)$. Then $J u_{b}=J u_{1}-J u_{2}$ and $J_{b} v=J v_{1}-J v_{2}$ and $J u_{1}, J v_{1} \in V_{1}(B)$ and $J u_{2}, J v_{2} \in V_{2}(B)$. Hence $A\left(J_{b} u, J_{b} v\right)=A\left(J u_{\mathrm{I}}, J v_{1}\right)+A\left(J u_{2}, J v_{2}\right)=A\left(u_{1}, v_{1}\right)+A\left(u_{2}, v_{2}\right)$. We can then 
define a Hermitian form $H_{b}$ on the complex vector space $V_{b}$ by

$$
H_{b}(u, v)=A\left(J_{b} u, v\right)+i A(u, v) .
$$

Then the imaginary part of $H_{b}$ is $A$ and we have $H_{b}\left(v_{k}, v_{j}\right)=A\left(J_{b} v_{k}, v_{j}\right)+$ $i A\left(v_{k}, v_{j}\right)=\varepsilon_{k} A\left(J v_{k}, v_{j}\right)=\varepsilon_{k}^{2} \delta_{k j}=\delta_{k j}$. This means that $B$ is an orthonormal basis of $V_{b}$ for the Hermitian form $H_{b}$ and in particular $H_{b}$ is positive definite and the decomposition (2) of $W$ is also orthogonal for $H_{b}$.

Let now

$$
E_{b}=V_{b} / L
$$

Then $H_{b}$ is a positive definite Riemann form for $E_{b}$ and hence $E_{b}$ is an abelian variety. Thus we have associated to a complex torus $E$ and a Riemann form $H$ of signature $(s, r)$ a family of polarized abelian varieties $\left(E_{b}, H_{b}\right)$ parametrized by $b \in \mathscr{B}=U(H) / K$.

We need the following lemma in the next section.

Lemma. We have

$$
H(u, v)=H_{b}(u, v) \quad \text { for } \quad u \in V_{1}(B)
$$

and

$$
H(u, v)=-H_{b}(v, u) \quad \text { for } \quad u \in V_{2}(B) .
$$

For we have $H_{b}(u, v)=A\left(J_{b} u, v\right)+i A(u, v)$ and $J_{b} u=J u$ or $J_{b} u=-J u$ according as $u \in V_{1}(B)$ or $u \in V_{2}(B)$.

\section{The cohomology group $H^{r}\left(E, F\left(H, \zeta^{\prime}\right)\right)$}

We associate to the Riemann form $H$ of signature $(s, r)$ for $E$ the factor $J_{H, \psi}$ defined by (1) and the line bundle $F(H, \psi)$ over $E$. For the cohomology groups of $F(H, \psi)$ we have the following theorem.

Theorem 1. (i) We have $H^{q}(E, F(H, \psi))=0$ for $q \neq r$.

(ii) Let $\left(z_{1}, \cdots, z_{n}\right)$ be the coordinates of the complex vector space $V$ determined by a privileged basis $B$ of $V$ for the Hermitian form $H$. Then $H^{r}(E, F(H, \psi))$ is identified with the ccmplex vector space of all $C^{\infty}$ functions $f$ in $V$ satisfying the following conditions:

1) $f$ is a differentiable theta function for the factor $J_{H, \psi}$; namely we have

$$
f(z+g)=J_{H, \psi}(z, g) \cdot f(z), z \in V, g \in L .
$$

2) $\frac{\partial f}{\partial \bar{z}_{k}}=0$ for $k \in[1, s]$

and

$$
\frac{\partial f}{\partial z_{s+j}}+\pi \bar{z}_{s+j} \cdot f=0 \quad \text { for } \quad j \in[1, r] .
$$


The assertion (i) in Theorem 1 is a well-known vanishing theorem due to Mumford. We shall give a proof of Theorem 1 in the Appendix 2 based on the harmonic theory.

We denote by $H(B)$ the space of $C^{\infty}$ functions $f$ on $W$ satisfying the above conditions (1) and (2) to make explicit its dependence of the condition (2) on the choice of the privileged basis $B$. We show that if $B$ and $B^{\prime}$ are equivalent, then we have $H(B)=H\left(B^{\prime}\right)$. In fact, let $\left(z_{1}^{\prime}, \cdots, z_{n}^{\prime}\right)$ be the coordinates of $V$ determined by $B^{\prime}$. Then we have

$$
z_{i}^{\prime}=\sum_{j=1}^{s} a_{i j} z_{j} \quad(i=1, \cdots, s)
$$

and

$$
z_{s+i}^{\prime}=\sum_{j=1}^{r} b_{i} z_{s+j} \quad(i=1, \cdots, r)
$$

where the matrices $\left(a_{i j}\right)$ and $\left(b_{i j}\right)$ are both unitary. We get

$$
\frac{\partial f}{\partial \bar{z}_{k}}=\sum_{i=1}^{s} a_{i k} \frac{\partial f}{\partial \bar{z}_{i}^{\prime}} \quad(k=1, \cdots, s)
$$

and

$$
\frac{\partial f}{\partial z_{s+k}}+\pi \bar{z}_{s+k} f=\sum_{i=1}^{r} b_{i k} \frac{\partial f}{\partial z_{s+i}^{\prime}}+\pi\left(\sum_{i=1}^{r} \bar{b}_{k i}^{\prime} \bar{z}_{s+1}^{\prime}\right) f,
$$

where $\left(b_{k i}^{\prime}\right)$ is the inverse matrix of $\left(b_{k i}\right)$. Since $\left(b_{k i}\right)$ is unitary, we have $\left(b_{k i}^{\prime}\right)=$ ${ }^{t}\left(\bar{b}_{k i}\right)$ and hence $\bar{b}_{k i}^{\prime}=b_{i k}$ Hence we get

$$
\frac{\partial f}{\partial z_{s+k}}+\pi \bar{z}_{s+k} f=\sum_{i=1}^{r} b_{i k}\left(\frac{\partial f}{\partial z_{s+i}^{\prime}}+\pi \bar{z}_{s+i}^{\prime} f\right)
$$

From $\left(^{*}\right)$ and $(* *)$ we get $H(B)=H\left(B^{\prime}\right)$. Hence we can denote the space of $C^{\infty}$ functions $f$ satisfying (1) and (2) by $H(b), b \in \mathscr{S}$.

Consider now the family of polarized abelian varieties $\left(E_{b}, H_{b}\right)(b \in B)$ defined in $\S 1$. We have the factor $J_{H_{b}, \psi}: L \times V_{b} \rightarrow C^{*}$ defined by

$$
J_{H_{b}, \psi}(g, u)=\psi(g) \varepsilon\left[\frac{1}{2 i} H_{b}(u, g)+\frac{1}{4 i} H_{b}(g, g)\right]
$$

where $g \in L$ and $u \in V_{b}$; this is because the imaginary part of $H_{b}$ is equal to $A$ for any $b$. Let $F\left(H_{b}, \psi\right)$ be the line bundle over $E_{b}$ associated with the factor $J_{H_{b}, \psi}$. Since $H_{b}$ is positive definite, we have $H^{q}\left(E_{b}, F\left(H_{b}, \psi\right)\right)=0$ for $q \neq 0$ and $H^{0}\left(E_{b}, F\left(H_{b}, \psi\right)\right)$ is identified with the complex vector space of all holomorphic theta functions on $V_{b}$ for the factor $J_{H_{b}, \psi}$.

Let $p_{i}: W \rightarrow V_{i}(B)(i=1,2)$ be the projection of $W$ onto $V_{i}(B)$ with respect 
to the decomposition (2) of $W$ and let

We have

$$
\phi_{b}(u)=\exp \left[-\pi H_{b}\left(p_{2}(u), p_{2}(u)\right)\right] .
$$

where

$$
\phi_{b}(u+g)=\phi_{b}(u) \exp L(u, g), u \in W, g \in L,
$$

$$
L(u, g)=-\pi\left[H_{b}\left(p_{2}(u), p_{2}(g)\right)+H_{b}\left(p_{2}(g), p_{2}(u)\right)+H_{b}\left(p_{2}(g), p_{2}(g)\right)\right] .
$$

Let $\theta$ be a holomorphic theta function on $V_{b}$ for the factor $J_{H_{b}, \psi}$ and let

$$
f=\phi_{b} \cdot \theta
$$

We show that the function $f$ satisfies the conditions (1) and (2) in Theorem 1 , i.e. $f \in H(b)$. We have

$$
f(u+g)=f(u) \psi(g) \exp \left[L(u, g)+\pi H_{b}(u, g)+\frac{\pi}{2} H_{b}(g, g)\right] .
$$

Since the decomposition (2) is orthogonal for $H_{b}$ we get

$$
\begin{aligned}
& \pi H_{b}(u, g)+\frac{\pi}{2} H_{b}(g, g)=\pi H_{b}\left(p_{1}(u), p_{1}(g)\right)+\pi H_{b}\left(p_{2}(u), p_{2}(g)\right)+\frac{\pi}{2} H_{b}\left(p_{1}(g), p_{1}(g)\right) \\
& +\frac{\pi}{2} H_{b}\left(p_{2}(g), p_{2}(g)\right) \text { and hence } L(u, g)+\pi H_{b}(u, g)+\frac{\pi}{2} H_{b}(g, g) \\
& =\pi\left[H_{b}\left(p_{1}(u), p_{1}(g)\right)-H_{b}\left(p_{2}(g), p_{2}(u)\right)\right]+\frac{\pi}{2}\left[H_{b}\left(p_{1}(g), p_{1}(g)\right)-H_{b}\left(p_{2}(g), p_{2}(g)\right)\right] .
\end{aligned}
$$

From Lemma at the end of $\S 1$ and from the orthogonality of the decomposition (2) for $H$ we see that the left hand side of the above equality is equal to $\pi H(u, g)+$ $\frac{\pi}{2} H(g, g)$. Hence we get $f(u+g)=f(u) \cdot \psi(g) \exp \left[\pi H(u, g)+\frac{\pi}{2} H(g, g)\right]=$ $f(u) J_{H}, \psi(g, u)$ which shows that $f$ is a differentiable theta function for the factor $J_{H, \psi}$.

Now let $B$ be any privileged basis representing $b$ and let $\left(z_{1}, \cdots, z_{n}\right)$ be the coordinates of $V$ determined by $B$. Then $B$ is also an orthonormal basis of $V_{b}$ for the Hermitian form $H_{b}$ and let $\left(w_{1}, \cdots, w_{n}\right)$ be the coordinates of $V_{b}$ determined by $B$. Then as functions on $W$ we have

$$
\begin{aligned}
& z_{i}=w_{i} \text { for } \quad i \in[1, s], \\
& \bar{z}_{s+i}=w_{s+i} \text { for } i \in[1, r] .
\end{aligned}
$$

Since $\theta$ is a holomorphic function on $V_{b}$ we have

$$
\frac{\partial \theta}{\partial \bar{w}_{k}}=0, \text { for } k \in[1, n]
$$


and hence

$$
\frac{\partial \theta}{\partial \bar{z}_{i}}=0, \quad i \in[1, s] ; \frac{\partial \theta}{\partial z_{s+i}}=0, \quad i \in[1, r]
$$

If $u=\sum_{k=1}^{n} z_{k} v_{k}$, then $p_{2}(u)=\sum_{i=1}^{r} z_{s+i} v_{s+i}$ and hence $H_{b}\left(p_{2}(u), p_{2}(u)\right)=\sum_{i=1}^{r}\left|z_{s+i}\right|^{2}$ and so

$$
\phi_{b}=\exp \left[-\pi \sum_{i=1}^{r}\left|z_{s+i}\right|^{2}\right] .
$$

We see easily that we have $\frac{\partial f}{\partial \bar{z}_{i}}=0$ for $i \in[1, s]$ and $\frac{\partial f}{\partial \bar{z}_{s+i}}+\pi \bar{z}_{s+i} f=0$ and hence $f$ belongs to $H(b)$. Analogously we can see that if $f$ is a function belonging to $H(b)$, then the function $\theta$ defined by $\theta(u)=f(u) \cdot \phi_{b}(u)^{-1}$ is a holomorphic theta function on $V_{b}$ for the factor $J_{H_{b} \psi}$ and moreover the map $f \rightarrow \theta$ defines a bijection of $H(b)$ onto the space $H^{0}\left(E_{b}, F\left(H_{o}, \psi\right)\right)$ of holomorphic theta functions on $V_{b}$ for the factor $J_{H_{b}, \psi}$. Since $H(b)$ is canonically isomorphic to $H^{r}(E, F(H, \psi))$ by Theorem 1 , we obtain the following theorem.

Theorem 2. Let $H$ be a Riemann form of signature $(s, r)$ for a complex torus $E$ and let $F(H, \psi)$ be the holomorphic line bundle over $E$ associated with the factor $J_{H, \psi}$ defined by $(1)$. Let $\left(E_{b}, H_{b}\right)$ and $\left(F\left(H_{b}, \psi\right)\right)$ be the family of polarized abelian varieties and the family of line bundles over each $E_{b}$ parametrized by $b \in \mathcal{B}$. Then there exists a canonical isomorphism of $H^{r}(E, F(H, \psi))$ onto $H^{0}\left(E_{b}, F\left(H_{b}, \psi\right)\right)$.

In particular, we have

$$
\operatorname{dim} H^{r}(E, F(H, \psi))=\operatorname{dim} H^{0}\left(E_{o}, F\left(H_{b}, \psi\right)\right)
$$

and since the imaginary part of $H_{b}$ is equal to the imaginary part $A$ of $H$, we have $\operatorname{dim} H^{0}\left(E_{b}, F\left(H_{b}, \psi\right)\right)=e_{1}, \cdots, e_{n}$, where $e_{1}, \cdots, e_{n}$ are the elementary divisors of the integral valued alternating form $A$ on $L \times L$. Thus we get also

$$
\operatorname{dim} H^{r}(E, F(H, \psi))=e_{1} \cdots e_{n} .
$$

Let $N+1=\operatorname{dim} H^{r}\left(E, F(H, \psi)\right.$ and let $\left(f_{0}, f_{1}, \cdots, f_{N}\right)$ be a basis of the complex vector space $H(b)$ which is canonically isomorphic to $H^{r}(E, F(H, \psi)$. The map $u \rightarrow\left[f_{0}(u): \cdots: f_{N}(u)\right]$ defines a differentiable map $\tilde{\Phi}$ from $W$ into the complex projective space $P^{N}$. Since each $f_{i}$ is a differentiable theta function on $W$ for the factor $J_{H, \psi}$, the map $\tilde{\Phi}$ defines a map $\Phi$ from $E=V \mid L$ into $P^{N}$.

Let $\theta_{i}=\phi_{b} \cdot f_{i}$ for $i \in[0, N]$. Then we have:

$$
\left[\theta_{0}(u): \theta_{1}(u): \cdots: \theta_{N}(u)\right]=\left[f_{0}(u): f_{1}(u): \cdots: f_{N}(u)\right] \text {. }
$$

It follows from this that $\Phi$ defines a holomorphic map from $E_{b}=V_{b} / L$ to $P^{N}$. We 
may assume without loss of generality that $\Phi$ is a holomorphic imbedding (this can be achieved by replacing $H$ by $3 H$ and $\psi$ by $\psi^{3}$ ). Then $\Phi$ defines a differentiable imbedding of $E$ into $P^{N}$. Let $\left(z_{1}, \cdots, z_{n}\right)$ be the coordinates on $V$ corresponding to a privileged basis of $V$ for $H$. Then these coordinates define local coordinates of the complex torus $E$ at each point of $E$. Since $\Phi$ is holomorphic as a map from $E_{b}$ into $P^{N}$, we see that $\Phi$ is holomorphic in $z_{1}, \cdots, z_{s}$ and anti-holomorphic in $z_{s+1}, \cdots, z_{n}$. Thus we get the following theorem.

Theorem 3. Let $H$ be a Riemann form of signature $(s, r)$ for a complex torus $E$. Then the cohomology group $H^{r}\left(E, F\left(3 H, \psi^{3}\right)\right)$ of the holomorphic line bundle $F\left(3 H, \psi^{3}\right)$ defines a differentiable imbedding of $E$ into the complex projective space $P^{N}$ with $N+1=\operatorname{dim} H^{r}\left(E, F\left(3 H, \psi^{3}\right)\right)$ which is holomorphic in $z_{1}, \cdots, z_{s}$ and antiholomorphic in $z_{s+1}, \cdots, z_{n}$, where $\left(z_{1}, \cdots, z_{n}\right)$ are the coordinates of the complex vector space $V$ determined by a privileged basis for $H$.

Appendix 1. We give here an example of a complex 2-torus which is not an abelian variety and which admits a Riemann form or signature $(1,1)$. Let

$$
\omega_{1}=\left(\begin{array}{l}
1 \\
0
\end{array}\right), \quad \omega_{2}=\left(\begin{array}{l}
0 \\
1
\end{array}\right), \quad \omega_{3}=\left(\begin{array}{l}
i \sqrt{2} \\
i \sqrt{3}
\end{array}\right), \quad \omega_{4}=\left(\begin{array}{c}
i \sqrt{3} \\
-i \sqrt{5}
\end{array}\right) .
$$

These vectors are linearly independent over $\boldsymbol{R}$ and they generate a lattice $L$ of $\boldsymbol{C}^{2}$.

The matrix $J_{0}$ of the complex structure of $C^{2}$ with respect to the basis $\left\{\omega_{1}, \omega_{2}, \omega_{3}, \omega_{4}\right\}$ of $\boldsymbol{C}^{2}$ over $\boldsymbol{R}$ is of the form

where

$$
J_{0}=\left(\begin{array}{cc}
0 & J_{1} \\
J_{2} & 0
\end{array}\right)
$$

and

$$
J_{1}=\left(\begin{array}{rr}
-\sqrt{2} & -\sqrt{3} \\
-\sqrt{3} & \sqrt{5}
\end{array}\right)
$$

$$
J_{2}=\frac{1}{d}\left(\begin{array}{rr}
-\sqrt{5} & -\sqrt{3} \\
-\sqrt{ } \overline{3} & \sqrt{2}
\end{array}\right), \quad d=-\sqrt{10}-3 .
$$

Let $A$ be an alternating $\boldsymbol{R}$-bilinear form on $\boldsymbol{C}^{2} \times \boldsymbol{C}^{2}$ which is integral valued on $L \times L$ and let $A_{0}$ be the matrix of $A$ with respect to the basis $\left\{\omega_{i}\right\}$ and write

$$
A_{0}=\left(\begin{array}{rr}
P_{1} & P_{2} \\
-{ }^{t} P_{2} & P_{3}
\end{array}\right) \quad P_{1}=\left(\begin{array}{rr}
0 & p \\
-p & 0
\end{array}\right) \quad P_{3}=\left(\begin{array}{rr}
0 & q \\
-q & 0
\end{array}\right),
$$

where $p$ and $q$ are integers and $P_{2}$ is an integral $2 \times 2$ matrix. 
The alternating form $A$ is the imaginary part of a Riemann form if and only if the $\boldsymbol{R}$-bilinear form $S$ on $\boldsymbol{C}^{2} \times \boldsymbol{C}^{2}$ defined by $S(u, v)=A(i u, v)$ is symmeiric and non-degenerate. Let $S_{0}$ be the matrix of $S$ with respect to $\left\{\omega_{i}\right\}$. Then we have $S_{0}={ }^{t} J_{0} \cdot A_{0}$. We see easily that the condition that $S_{0}$ is symmetric is equivalent to the set of the following three conditions: (a) $P_{1} J_{1}=-{ }^{t} J_{2} P_{3}$; (b) $P_{2} J_{2}$ is symmetric; (c) ${ }^{t} P_{2} J_{1}$ is symmetric. The conditions (b) and (c) are both equivalent to the single condition that $P_{2}$ is to be of the form

$$
P_{2}=\left(\begin{array}{ll}
a & 0 \\
0 & a
\end{array}\right) \quad a \in Z \text {. }
$$

The condition (a) is equivalent to the condition $p d=q$, where $d=-\sqrt{10}-3$ and $p$ and $q$ are integers and so (a) is equivalent to the condition $p=q=0$.

Thus we reached the conclusion that $S_{0}$ is symmetric if and only if $A_{0}$ is the form

$$
A_{0}=\left(\begin{array}{cc}
0 & a 1_{2} \\
-a 1_{2} & 0
\end{array}\right)
$$

where $a \neq 0$ is an integer and $1_{2}$ denote the $2 \times 2$ unit matrix. Then $S_{0}$ takes the form

$$
S_{0}=\left(\begin{array}{cc}
-a J_{2} & 0 \\
0 & a J_{1}
\end{array}\right)
$$

and $S_{0}$ is a non-singular matrix. However $S_{0}$ is not definite because the symmetric matrix $J_{1}$ is not definite. Thus $A$ can be the imaginary part of a Riemann form if and only if $A_{0}$ is of the form $\left(^{*}\right)$ and when this is the case, the corresponding Riemann form is not definite but of signature $(1,1)$. Hence $E=\boldsymbol{C}^{2} / L$ provides an example of a complex torus which is not an abelian variety and which admits a Riemann form of signature $(1,1)$.

Appendix 2. Since the second assertion in Theorem 1 is an essential part of this article we give a proof of Theorem 1 in this appendix.

Let $H$ be a Riemann form of signature $(s, r)$ for a complex torus $E=V \mid L$ and $J_{H, \psi}$ the factor defined by (1) and $F(H, \psi)$ the holomorphic line bundle associated with $J_{H, \psi}$. Let $D^{q}$ be the vector space of all $F(H, \psi)$-valued differential forms of type $(0, q)$ on $E$. Then the cohomology group $H(E, F(H, \psi))$ of $E$ with coefficient in the sheaf of germs of holomorphic sections of $F(H, \psi)$ is isomorphic to the cohomology group of the complex $D=\sum_{i=0}^{n} D^{q}$, where the coboundary operator is given by $d^{\prime \prime}($ or $\bar{\partial})$. On the other hand, there exists a canonical identification of an $F(H, \psi)$-valued $(0, q)$-form $\alpha$ on $E$ with $a(0, q)$ form $\phi$ on $V$ (of class $C^{\infty}$ ) satisfying the condition that 


$$
T_{g}^{*} \varphi=J_{H, \psi}(g, \cdot) \varphi
$$

for $g \in L$, where $T_{g}$ denotes the translation of $V$ by $g$. Then $d^{\prime \prime} \varphi$ also satisfies the same condition (*) and $d^{\prime \prime} \alpha$ is identified with $d^{\prime \prime} \varphi$. Denote by $A^{q}$ the vector space of all $(0, q)$-form on $V$ (of class $C^{\infty}$ ) satisfying the condition (*). Then the cohomology group $H(E, F(H, \psi))$ is isomorphic to the cohomology group of the complex $A=\sum_{q=0}^{n} A^{q}$, where the coboundary operator is given by $d^{\prime \prime}$. Notice that $A^{0}$ is the vector space of all differentiable theta functions on $V$. Let $\left(z_{1}, \cdots, z_{n}\right)$ be coordinates on $V$. Then a $(0, q)$-form is expressed uniquely in the form

$$
\varphi=\frac{1}{q !} \sum_{J} \varphi_{J} d \bar{z}_{J},
$$

where $J=\left(j_{1}, \cdots, j_{q}\right)$ is a multi-index and each $\varphi_{J}$ is alternating in the indices and $d \bar{z}_{J}=d \bar{z}_{j_{1}} \wedge \cdots \wedge d \bar{z}_{j_{q}}$. Since $d \bar{z}_{J}$ is invariant by translation, $\varphi$ satisfies the condition $\left(^{*}\right)$ if and only if each component $\varphi_{J}$ belongs to $A^{0}$.

Lemma 1. Let $f, g \in A^{0}$ and let $\langle f, g\rangle$ be defined by

$$
\langle f, g\rangle(u)=f(u) g(u) \exp [-\pi H(u, u)]
$$

for $u \in V$. Then the function $\langle f, g\rangle$ is invariant by the translation $T_{g}$ for any $g \in L$.

We can verify the lemma by a straightforward computation.

We may consider $\langle f, g\rangle$ as function on the torus $E=V \mid L$.

Corollary of Lemma 1. If $f, g \in A^{0}$, then

$$
|f(u)||g(u)|<C \exp \pi H(u, u)
$$

for any $u \in V$, where $C$ is a positive constant.

Let us choose a positive definite Hermitian form $G$ and let

$$
G=\sum_{i, j} g_{i j} z_{i} \bar{z}_{j} .
$$

Let

$$
d V=\left(\frac{i}{2}\right)^{n} \operatorname{det}\left(g_{i j}\right) d z_{1} \wedge d \bar{z}_{1} \wedge \cdots \wedge d z_{n} \wedge d \bar{z}_{n}
$$

the volume element on $V$ determined by $G$. The volume element $d V$ is invariant by translation and so it defines a translation invariant volume element $d v$ on $E$ such that $\pi^{*} d v=d V$, where $\pi: V \rightarrow E$ is the canonical projection. We define the inner product $(f, g)$, where $f, g \in A^{0}$, by 


$$
(f, g)=\int_{P}\langle f, g\rangle d V,
$$

where $P$ is a fundamental paralleltop for the lattice $L$, or equivalently by

$$
(f, g)=\int_{\Xi}\langle f, g\rangle d v
$$

regarding $\langle f, g\rangle$ as function on $E$.

Let us write

$$
H=\sum_{i, j} h_{i j} z_{i} \bar{z}_{j}
$$

and introduce covariant derivations $D_{i}^{\prime}, D_{i}^{\prime \prime}(i=1, \cdots, n)$ by the formula

$$
\begin{aligned}
& \left(D_{i}^{\prime} f\right)(z)=\frac{\partial f}{\partial z_{i}}(z)-\pi\left(\sum_{k} h_{i k} \bar{z}_{k}\right) f(z), \\
& \left(D_{i}^{\prime \prime} f\right)(z)=\frac{\partial f}{\partial \bar{z}_{i}}(z) .
\end{aligned}
$$

We can show without difficulty that if $f \in A^{0}$, then we have $D_{i}^{\prime} f, D_{i}^{\prime \prime} f \in A^{0}$ for $i=1, \cdots, n$. We have also the following formulas:

$$
\begin{aligned}
& \left\langle D_{i}^{\prime} f, g\right\rangle+\left\langle f, D_{i}^{\prime \prime} g\right\rangle=\frac{\partial}{\partial z_{i}}\langle f, g\rangle, \\
& \left\langle D_{i}^{\prime \prime} f, g\right\rangle+\left\langle f, D_{i}^{\prime} g\right\rangle=\frac{\partial}{\partial \bar{z}_{i}}\langle f, g\rangle .
\end{aligned}
$$

Integralating both sides of the equalities and using the Green's theorem we obtain

$$
\begin{aligned}
& \left(D_{i}^{\prime} f, g\right)+\left(f, D_{i}^{\prime \prime} g\right)=0, \\
& \left(D_{i}^{\prime \prime} f, g\right)+\left(f, D_{i}^{\prime} g\right)=0,
\end{aligned}
$$

where $f, g \in A^{0}$ and $i \in[1, n]$.

Denote by $g^{i j}$ the $(i, j)$-entry of the inverse matrix of the Hermitian matrix $\left(g_{i j}\right)$ and let

$$
g^{I J}=g^{i_{1} j_{1} \ldots .} g^{i_{q} j_{q}}
$$

where $I=\left(i_{1}, \cdots, i_{q}\right)$ and $J=\left(j_{1}, \cdots, j_{q}\right)$.

For $\varphi, \psi \in A^{q}$, we define the function $\langle\varphi, \psi\rangle$ by

$$
\langle\varphi, \psi\rangle=\frac{1}{q !} \sum_{T, J} g^{I J}\left\langle\varphi_{I}, \psi_{J}\right\rangle
$$

Then $\langle\varphi, \psi\rangle$ is invariant by the translation $T_{g}(g \in L)$ and we define the inner 
product $(\varphi, \psi)$ by

$$
(\varphi, \psi)=\int_{P}\langle\varphi, \psi\rangle d V=\int_{\forall}\langle\varphi, \psi\rangle d v .
$$

There exists the adjoint operator $\mathfrak{D}$ for the operator $d^{\prime \prime}: A^{q} \rightarrow A^{q+1}$ so that we have

for $\varphi \in A^{q}$ and $\psi \in A^{q+1}$

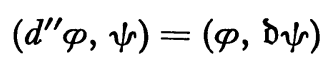

We define the Laplacian $\square$ by

$$
\square=d^{\prime \prime} \mathrm{D}+\mathrm{D} d^{\prime \prime}
$$

Then $\square$ is an operator from $A^{q}$ to $A^{q}$ for all $q$ and a $(0, q)$-form $\varphi \in A^{q}$ is said to be harmonic if $\square \varphi=0$. Each element of the cohomology group $H^{q}(A)$ of the complex $A$ is represented by a unique harmonic form. In this sense we can say that each element of the cohomology group $H^{q}(E, F(H, \psi)$ is represented by a unique harmonic form. Thus we may identify $H^{q}(E, F(H, \psi))$ with the vector space of all harmonic forms in $A^{q}$. We now introduce the following notation. For $I=\left(i_{1}, \cdots, i_{q+1}\right), I_{u}$ will denote the multi-index $\left(i_{1}, \cdots, \hat{i}_{u}, \cdots, i_{q+1}\right)$, where the index $i_{u}$ under $\wedge$ is omitted. We also introduce the operator $D^{\prime i}$ by

$$
D^{\prime i}=\sum_{j} g^{i j} D_{j}^{\prime}
$$

We can prove the following three formulas.

A) Let $\varphi \in A^{q}$. Then the components $\left(d^{\prime \prime} \varphi\right)_{I}, I=\left(i_{1}, \cdots, i_{q+1}\right)$ of $d^{\prime \prime} \varphi \in A^{q+1}$ is given by the formula

$$
\left(d^{\prime \prime} \varphi\right)_{I}=\sum_{u=1}^{q+1}(-1)^{u+1} D_{i_{u}}^{\prime \prime} \varphi_{I_{u}}
$$

B) Let $\psi \in A^{q+1}$. Then the component $(D \psi)_{J}, J=\left(j_{1}, \cdots, j_{q}\right)$, of $D \psi$ is given by the formula

$$
\left(\mathcal{D} \psi_{J}=-\sum_{j=1}^{n} D^{\prime j} \psi_{j J}\right.
$$

where $j J=\left(j, j_{1}, \cdots, j_{q}\right)$.

C) Let $\varphi \in A^{q}$. Then the component $(\square \varphi)_{I}$ of $\square \varphi \in A^{q}$ is given by the formula

$$
(\square \varphi)_{I}=-\left(\sum_{i=1}^{n} D^{\prime i} D_{i}^{\prime \prime}\right) \cdot \varphi_{I}+\pi \sum_{u=1}^{q}(-1)^{u+1} \sum_{i=1}^{n}\left(\sum_{k=1}^{n} g^{i k} h_{k i_{u}}\right) \varphi_{i I_{u}}
$$

where $i I_{u}=\left(i, i_{1}, \cdots, \hat{i}_{u}, \cdots, i_{q}\right)$.

We omit the proof of these formulas. Similar formulas had been proved in [1] in a somewhat different context, but the proof can be carried out quite 
similarly.

Up to this point the choices of the coordinates $\left(z_{1}, \cdots, z_{n}\right)$ and the positive definite Hermitian form $G=\sum_{i, j} g_{i}, z_{i} \bar{z}_{j}$ are arbitrary. From now on we choose privileged coordinates $\left(z_{1}, \cdots, z_{n}\right)$ for the Hermitian form $H$ so that we have

$$
H=\sum_{i=1}^{s}\left|z_{i}\right|^{2}-\sum_{i=1}^{r}\left|z_{s+i}\right|^{2}
$$

and hence we have $h_{i j}=0$ for $i \neq j$ and $H_{i i}=\varepsilon_{i}$ (the symbol $\varepsilon_{i}$ being defined in $\S 1$ ). We choose $G$ such that

$$
G=\frac{1}{a}\left(\left|z_{1}\right|^{2}+\cdots+\left|z_{s}\right|^{2}\right)+\left|z_{s+1}\right|^{2}+\cdots+\left|z_{n}\right|^{2},
$$

where $a>0$ (cf. [4]). Then we have $g^{i j}=0$ for $i \neq i$ and

Then we have

$$
g^{i i}= \begin{cases}a & \text { for } i \in[1, s] \\ 1 & \text { for } i \in[s+1, n]\end{cases}
$$

$$
\sum_{k} g^{i k} h_{k j}=\left\{\begin{aligned}
0, & i \neq j \\
a, & i=j \text { and } i \in[1, s] \\
-1, & i=j \text { and } i \in[s+1, n]
\end{aligned}\right.
$$

Let

$$
\alpha_{i}=\left\{\begin{aligned}
a & \text { for } i \in[1, s] \\
-1 & \text { for } i \in[s+1, n]
\end{aligned}\right.
$$

Then we have $\sum_{i=1}^{n}\left(\sum_{k=1}^{n} g^{i k} h_{k i_{u}}\right) \varphi_{i I_{u}}=(-1)^{u+1} \alpha_{i_{u}} \varphi_{I}$ and we get

$$
(\square \varphi)_{I}=-\left(\sum_{i} D^{\prime i} D_{i}^{\prime \prime}\right) \varphi_{I}+\pi\left(\sum_{u=1}^{q} \alpha_{i_{u}}\right) \varphi_{I},
$$

where

$$
D^{\prime i}=g^{i i} D_{i}^{\prime} \quad \text { (not summed). }
$$

From this we obtain

$$
\left((\square \varphi)_{I}, \varphi_{I}\right)=\sum_{i=1}^{n} g^{i i}\left(D_{i}^{\prime \prime} \varphi_{I}, D_{i}^{\prime \prime} \varphi_{I}\right)+\pi\left(\sum_{u=1}^{q} \alpha_{i_{u}}\right)\left(\varphi_{I}, \varphi_{I}\right) .
$$

Since the first term of the right hand side is non-negative, we get

$$
\left((\square \varphi)_{I}, \varphi_{I}\right) \geqq \pi \cdot \alpha(I) \cdot\left(\varphi_{I}, \varphi_{I}\right) .
$$

where we put

$$
\alpha(I)=\sum_{u=1}^{q} \alpha_{i_{u}}
$$


Let us denote by $N$ (resp. $M$ ) the number of indices $i_{u}$ such that $i_{u} \leq s$ (resp. $\left.i_{u}>s\right)$. Then by the definition of $\alpha_{k}$, we have

$$
\alpha(I)=a \cdot N-M \text {. }
$$

For the multi-index $I$ we may assume that these $q$ indices are distinct, otherwise we get $\varphi_{I}=0$. Suppose $q>r$. Then at least one of the indices $i_{u}$ must be less than or equal to $s$ and hence $N \geqq 1$. Then we get

$$
\alpha(I) \geqq a-r
$$

Choose $a$ such that $a>r$. Then we have $\alpha(I)>0$ for $q>r$.

Suppose the $\square \varphi=0$, where $\varphi \in A^{q}$ with $q>r$. Then

$$
0=\left(\left(\square \varphi_{I}\right), \varphi_{I}\right) \geqq \pi \alpha(I) \cdot\left(\varphi_{I}, \varphi_{I}\right)
$$

with $\alpha(I)>0$. Hence we must have $\left(\varphi_{I}, \varphi_{I}\right)=0$ and hence $\varphi_{I}=0$ for any $I$ and this means $\varphi=0$. This shows that $H^{q}(A)=0$ and hence $H^{q}(E, F(H, \psi))=0$ for $q>r$.

On the other hand, by the Serre duality, we have

$$
H^{q}(E, F(H, \psi)) \simeq H^{n-q}\left(E, K \otimes F^{*}\right),
$$

where $K$ is the canonical line bundle of $E$ and $F^{*}$ is the dual of $F(H, \psi)$. It is easy to see that $F^{*} \cong F\left(-H, \varphi^{-1}\right)$ and $-H$ is of signature $(r, s)$. Moreover since $E$ is a complex torus, $K$ is a trivial bundle and so we get

$$
H^{q}(E, F(H, \psi)) \cong H^{n-q}\left(E, F\left(-H, \varphi^{-1}\right)\right) .
$$

Since $-H$ is of signature $(r, s)$, we get from what we have already proved that $H^{n-q}\left(E, F\left(-H, \varphi^{-1}\right)\right)=0$ whenever $n-q>s$ or whenever $n-s=r>q$. Thus we get $H^{q}(E, F(H, \psi))=0$ for $q<r$ and these prove the first assertion in Theorem 1 .

Consider now that case $q=r, \varphi \in A^{r}$ and $\square \varphi=0$. Even in this case we get $\alpha(I) \geqq a-r>0$ except in the case where all of the $r$ indices in $I$ are greater than $s$, namely except in the case where $I$ is a permutation of $(s+1, \cdots, n)$. Then we get $\varphi_{I}=0$ for each $I$ which is not a permutation of $(s+1, \cdots, n)$ and $\varphi$ is of the form

$$
\varphi=f d \bar{z}_{s+1} \wedge \cdots \wedge d \bar{z}_{n}
$$

where $f=\varphi_{s+1, \cdots, n}$.

Conversely let $\varphi$ be a $(0, r)$-form on $V$ of the type (**) belonging to $A^{r}$. Then $f \in A^{0}$ and we have

$$
d^{\prime \prime} \varphi=0 \rightleftharpoons \frac{\partial f}{\partial \bar{z}_{i}}=0 \quad \text { for } \quad i \in[1, s]
$$

and 


$$
(\delta \varphi)_{I}=-\sum_{i=1}^{n} g^{i i} D_{i}^{\prime} \varphi_{i I}
$$

where $I=\left(i_{1}, \cdots, i_{r-1}\right)$. If $I \neq(s+1, \cdots, \hat{u}, \cdots, n)$ for some $u,(i, I)$ cannot be a permutation of $(s+1, \cdots, n)$ and $\varphi_{i I}=0$ and hence $(\emptyset \varphi)_{I}=0$. If $I=(s+1, \cdots$, $\hat{u}, \cdots, n$ ) for some $u$, then

Therefore we have

$$
\left(\grave{\varphi)_{I}}= \pm g^{u u} D_{u}^{\prime} f\right.
$$

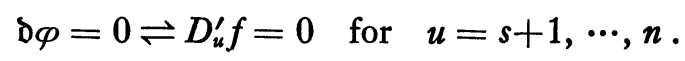

It follows from our definition of the operator $D_{u}^{\prime}$ and from the fact $h_{i j}=\delta_{i j} \cdot \varepsilon_{j}$, we see that

$$
D_{u}^{\prime} f=\frac{\partial f}{\partial z_{u}}+\pi \bar{z}_{u} f
$$

We have thus proved that the space of harmonic $(0, r)$-form $\varphi$ in $A^{r}$ consists of all the $(0, r)$-form $\varphi$ on $V$ of the form

where

$$
\varphi=f d \bar{z}_{s+1} \wedge \cdots \wedge d \bar{z}_{n},
$$

1) $f$ is a differentiable theta function for the factor $J_{H, \psi}$,

2) $\frac{\partial f}{\partial \bar{z}_{i}}=0$ for $i \in[1, s]$

and

$$
\frac{\partial f}{\partial z_{i}}+\pi \bar{z}_{i} f=0 \text { for } i \in[s+1, n] .
$$

Then we can identify the cohomology group $H^{r}(A)$ with the vector space of functions $f$ satisfying the conditions 1 ) and 2) and this proves the second assertion in Theorem 1.

\section{UNIVERSITY OF NOTRE DAME}

\section{Bibliography}

[1] Y. Matsushima and S. Murakami: On vector bundle valued harmonic forms and automorphic forms on symmetric Riemannian manifolds, Ann. of Math. 78 (1963), 365-416.

[2] D. Mumford: Abelian varieties, Tata Inst. Studies in Math., Oxford Univ. Press, 1970.

[3] C.L. Siegel: Indefinite quadratische Formen und Funktionentheorie. I, Math. Ann. 124 (1951), 17-54.

[4] H. Umemura: Some results in the theory of vector bundles, Nagova Math. J. 52 (1973), 97-128. 
